\title{
NMA SURVEY OF HCN AND CO EMISSION FROM NEARBY ACTIVE GALAXIES
}

\author{
KOHNO KOTARO, KAWABE RYOHEI, SAKAMOTO KAZUSHI, \\ ISHIZUKI SUMIO AND BALTASAR VILA-VILARO \\ Nobeyama Radio Observatory \\ Minamimaki, Minamisaku, Nagano, 384-13, JAPAN
}

\begin{abstract}
In order to study the spatial variation of molecular gas properties and their relation to the nuclear activities of galaxies, we have conducted an imaging survey of $\mathrm{HCN}(1-0)$ and $\mathrm{CO}(1-0)$ emission from nearby active galaxies with the Nobeyama Millimeter Array. Here we present the results of three galaxies, NGC 3504 (Starburst nucleus), NGC 4736 (PostStarburst nucleus), and NGC 6951 (Seyfert nucleus with a circumnuclear star-forming ring).

In the observations of these three galaxies, we find that (1) the HCN distribution is significantly different from that of $\mathrm{CO}$, and that (2) the $\mathrm{HCN}$ emission spatially correlates better with the massive star-forming region than CO emission (Figure 1, 2(a), and 2(b)). For instance, in the PostStarburst nucleus of NGC 4736, we find a remarkable decrease of the HCN emission, despite of strong $\mathrm{CO}$ nuclear concentration. The upper limit $(2 \sigma)$ of the integrated intensity ratio $\mathrm{I}(\mathrm{HCN}) / \mathrm{I}(\mathrm{CO})$ within the central $r<150$ pc region is 0.04 , which is surprisingly low compared with the value in other galactic nucleus $(c f$. ratio $=0.08$ within $r<300 \mathrm{pc}$ in Milky Way, Jackson et al. 1996, ApJ, 456, 91). Presumably the dense molecular gas must have been consumed by a past starburst event in the nucleus. These results suggest a tight correlation between the gas density measured by the $\mathrm{I}(\mathrm{HCN}) / \mathrm{I}(\mathrm{CO})$ ratio and the star-forming activities in the central region of galaxies.

It seems that there exist a spatial anti-correlation between the $\mathrm{I}(\mathrm{HCN}) /$ $\mathrm{I}(\mathrm{CO})$ ratio and the gas velocity dispersion in NGC 6951 (Figure 2(c)). The ratio is not enhanced in the $\mathrm{CO}$ twin peaks which are interpreted as shock regions due to gas orbit crowding. These imply the importance of gravitational instability on the formation of dense molecular gas and successive massive starformation in this region.
\end{abstract}




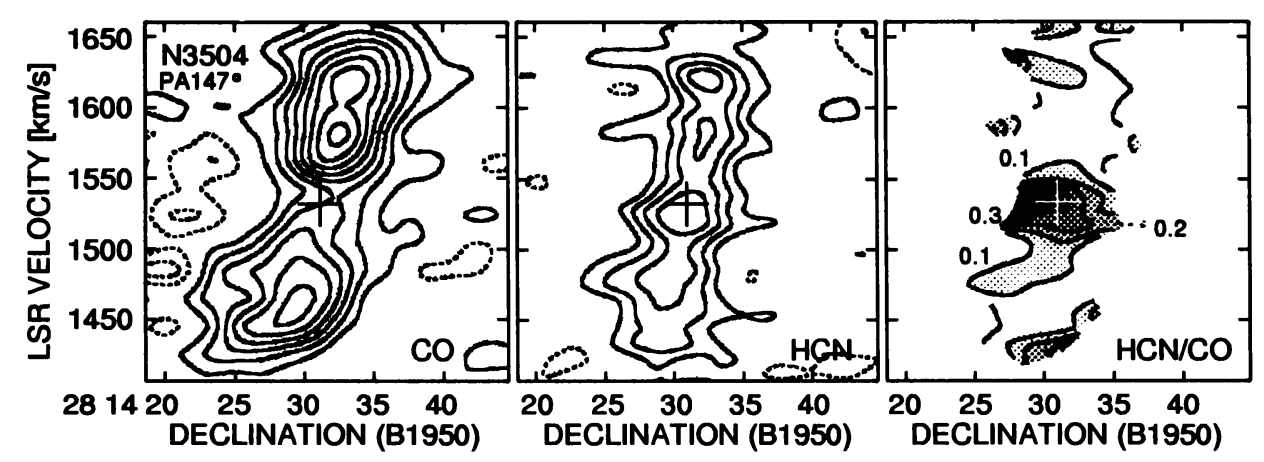

Figure 1. Position - velocity ( $p v)$ maps of $\mathrm{CO}$ and $\mathrm{HCN}$ along the major axis in NGC 3504 , and the brightness temperature ratio of them. Both the $\mathrm{CO}$ and $\mathrm{HCN}$ emissions are centrally concentrated in the integrated intensity maps, however, the $p v$ maps reveal a small CO depression near the systemic velocity (Sakamoto 1996, PhD thesis) while HCN peaks there. In this CO "hole", the ratio is as high as 0.3 , which is comparable to the ratio in the starburst region of M82 and NGC 253 (Shen \& Lo, 1995, ApJ, 445, L99; Paglione et al. 1995. ApJ, 454, L117). It is also evident that all of the HCN emission is distributed within the rigid-body rotating region, where massive star-formations occur (Kenney et al. 1993, ApJ, 418, 687)

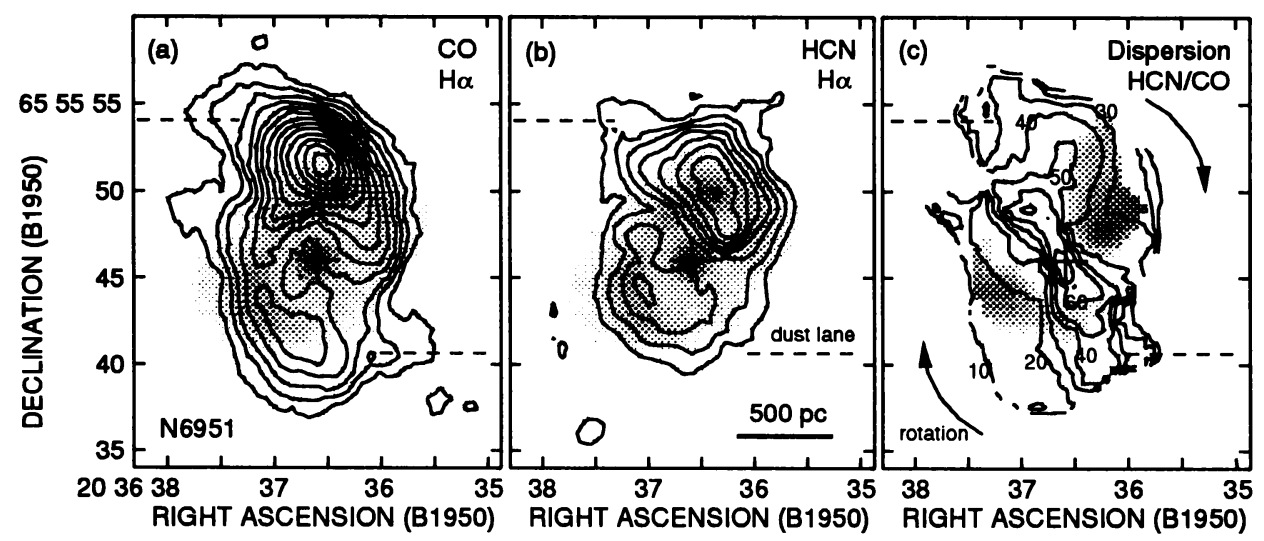

Figure 2. (a) and (b) show the CO and HCN contour maps of NGC 6951, superposed on $\mathrm{H} \alpha$ image (Wozniak et al. 1995, A\&A Suppl, 111, 115). In contrast to CO, most of the $\mathrm{HCN}$ emission comes from the $\mathrm{H} \alpha$ ring. Namely, the distribution of dense molecular gas is spatially well correlated with the massive star-forming region. (c) is a comparison between the $\mathrm{CO}$ velocity dispersion (contour) and the intensity ratio $\mathrm{I}(\mathrm{HCN}) / \mathrm{I}(\mathrm{CO})$ (halftone). This shows a spatial shift between $\mathrm{I}(\mathrm{HCN}) / \mathrm{I}(\mathrm{CO})$ ratio peaks and $\mathrm{CO}$ twin peaks where a large velocity dispersion is observed, although this dispersion contains the velocity gradient of the systematic rotation within a observing beam. The ratio toward this nucleus $(\mathrm{Sy} 2)$ is rather normal $(\sim 0.08)$, although very high ratios $(0.4-0.6)$ have been reported in some Seyfert nucleus such as NGC 1068 (Jackson et al. 1993, ApJ, 418, L13; Tacconi et al. 1994, ApJ, 426, L77) and M 51 (Kohno et al. 1996, ApJ, 461, L29). 